\title{
Reduced glycolytic reserve in isolated natural killer cells from Myalgic encephalomyelitis/chronic fatigue syndrome patients: A preliminary investigation
}

\author{
Thao Nguyen, ${ }^{1,2 *}$ Donald Staines, ${ }^{1,2}$ Samantha Johnston, ${ }^{1,2}$ Sonya Marshall-Gradisnik ${ }^{1,2}$
}

\begin{abstract}
Background: Myalgic Encephalomyelitis/Chronic Fatigue Syndrome (ME/CFS) is medically unexplained post-exertional fatigue associated with significant reduction in natural killer cell (NK) cytotoxicity activity. Cytotoxic activity relies on glycolytic flux and mitochondrial respiration to fulfill energetic cellular demands. While mitochondrial dysfunction has been reported in ME/CFS patients, no previous investigation has examined the bioenergetic profile of isolated NK cells from $\mathrm{ME} / \mathrm{CFS}$ patients.
\end{abstract}

Objective: This study was to determine the metabolic function in resting NK cells from ME/CFS patients.

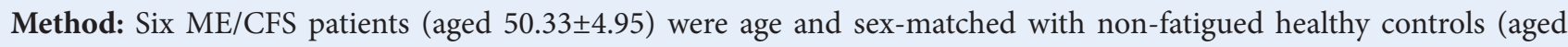
$50.00 \pm 5.04)$. Mitochondrial stress tests measured parameters of mitochondrial function in the NK cells including basal respiration, ATP production, proton leak, maximal respiration, spare respiratory capacity and bioenergetic health index. Glycolytic stress tests measured parameters of glycolytic function such as glycolytic reserve, glycolysis and glycolytic capacity in isolated NK cells from ME/CFS patients and healthy controls using an extracellular flux analyzer, Seahorse XFp.

Result: There was a significant reduction of glycolytic reserve in resting NK cells from ME/CFS patients $(0.6 \pm 0.07 \mathrm{mpH} /$ $\mathrm{min})$ compared with healthy control $(2.25 \pm 1.3 \mathrm{mpH} / \mathrm{min})$. Mitochondrial respiration in resting NK cells did not approach statistical significance between ME/CFS patients and healthy controls.

Conclusion: These findings suggest resting NK cells from ME/CFS patients have reduced ability to increase glycolytic flux to respond to high energetic demands for ATP production. Hence, the reduced glycolytic reserves we have identified in isolated resting isolated NK cells should be further investigated to assist in understanding ME/CFS pathogenesis.

Keywords: Natural killer cell, mitochondrial respiration, glycolytic reserve, glycolysis, glucose, ATP

From:

1 The National Centre for Neuroimmunology and Emerging Diseases, Menzies Health Institute Queensland, Griffith University, Gold Coast, QLD, Australia

${ }^{2}$ School of Medical Science, Griffith University, Gold Coast, QLD, Australia

\section{Introduction}

Myalgic Encephalomyelitis/Chronic Fatigue Syndrome (ME /CFS) is a multifactorial illness characterized by post-exertional fatigue that is accompanied by a range of neuro-immunological symptoms for example malaise and hyperalgesia. ${ }^{1}$ The etiology and underlying pathophysiological mechanism of ME/CFS are yet to be defined. A working hypothesis has been shown to relate immunological energy wastage and mitochondrial impairment in ME/CFS. ${ }^{2-4}$

\author{
* Corresponding author: \\ Thao Nguyen \\ Mail box 68, level 8, building G40, Griffith University \\ Parklands Drive, Southport, Gold Coast, Queensland, Australia, 4222 \\ E-mail: ncned@griffith.edu.au
}


to the multisystem nature of ME/CFS. Recently, Tomas et al (2017) measured metabolic profile in peripheral blood mononuclear cells (PBMC) in ME/CFS and demonstrated PBMC under stress have lower reserve capacity and maximal respiration function compare to healthy controls. ${ }^{9}$ Therefore, PBMCs are unable to increase respiration rate to compensate for the increase energy demand. PBMC are heterogeneous cell population containing monocytes, $\mathrm{T}$ cell, $\mathrm{B}$ cells and $\mathrm{NK}$ cells. To date there has been extensive research on NK cell receptors and effector function in ME/CFS patients but the role of metabolism on $\mathrm{NK}$ cell immune function remains to be elucidated.

In particular, the immune system utilizes energy for pathogen clearance, activation of inflammation and autoimmune responses. The most consistent immunological finding is significantly reduced cytotoxic activity in Natural Killer (NK) cells. ${ }^{10,11}$ Cytotoxicity is desirable to help fight infection and kill transformed cells to prevent diseases but requires high energy consumption. In sickness behavior, the body undergoes energy conservation which priorities the energy towards immune cells to help fight off pathogens through the release of proinflammatory cytokines. Whereas, ME/CFS illness has been suggested to be associated with activation of inflammation and oxidative stress, and yet may not resolve infection. ${ }^{12}$

NK cells belong to the innate immune system and are able to adapt to the surrounding environment during an inflammatory response. NK cells have two subsets that are identified as ${\text { CD } 56^{\text {Bright }} \text { and CD56 }}^{\text {Dim }}$ NK cells. Peripheral CD56 ${ }^{\text {Dim }}$ NK cells have cytotoxic functions to detect and kill target cells that do not express MHC-I molecule. Additionally, CD56 $6^{\text {Bright }} \mathrm{NK}$ cells are immuno-regulatory and are predominantly engaged in cytokine production. In particular releasing IFN- $\gamma$ to help recruit other immune cells to the site of inflammation or infection. $^{13,14}$

Isolated NK cells from ME/CFS patients have also been shown to have reduced effector molecules, such as IFN- $\gamma$, granzyme and perforin which are essential for cytotoxic activity. ${ }^{10,11,15,16} \mathrm{NK}$ cells require glycolytic flux and mitochondrial respiration for energy production. ${ }^{17}$ However, NK cells do not have significant intracellular glycogen stores and are dependent on importing extracellular glucose to fulfill energetic demands. ${ }^{18}$ Resting NK cells have low levels of glycolytic flux, where glucose is converted into pyruvate and oxidized by the Krebs Cycle to produce carbon dioxide and 2 ATP molecules. ${ }^{19}$ Whereas, activated NK cells consume higher energy to carry out functional activities. ${ }^{19}$ Therefore the Krebs cycle consumes acetyl CoA to produce $\mathrm{NADH}^{+}$and $\mathrm{FADH}$, which is fed into the mitochondrial respiration pathway, where oxidation and production of protons are produced from electron transport chain (ETC) complex I and II. The protons flow towards the complex V to produce 36 ATP molecules. ${ }^{19}$ Hence, activated NK cells increase glucose receptors expression and glycolytic enzymes for glycolytic flux to fuel mitochondrial respiration as this is the most effective pathway for synthesis of effector molecules and perform degranulation. ${ }^{17} \mathrm{NK}$ cells oxidize pyruvate into lactate which is then removed from the cell to sustain increased demands of glycolytic flux. ${ }^{19}$ This step is essential as the accumulation of pyruvate may result in inhibition of the glycolytic pathway.
Therefore, the purpose of this preliminary study was to investigate the mitochondrial respiration (basal, spare respiration, proton leak and ATP production) and glycolytic function (glycolysis, glycolytic capacity, and glycolytic reserve) in isolated NK cells from ME/CFS patients compared with healthy controls to examine their possible role in the pathogenesis of $\mathrm{ME} / \mathrm{CFS}$.

\section{Materials and methods Subject characteristics}

This cross-sectional study consisted of six healthy controls (aged 50.00 \pm 5.04 years) which were age- and sex- matched with six ME/CFS patients (aged $50.33 \pm 4.95$ years). Participants were residents of the South-East Queensland region of Australia and recruited from a university research database. The inclusion of healthy participants included screening for any underlying illness and ME/CFS participants met both the Fukuda criteria and International Consensus Criteria ${ }^{1,20}$ and have previously reported reduced NK lysis activity. ${ }^{10,15}$ Both healthy control and ME/CFS groups were required to have no exclusionary medical conditions including major disease such as autoimmune disease, cardiovascular disease, primary psychiatric disorders, pregnancy, and smokers. Further exclusions were any potential medications that were immune regulators and hormone therapy. Participants were screened using routine pathology blood tests, including full blood count, electrolytes, erythrocyte sedimentation rate and high sensitive C-reactive protein. All participants completed the Fatigue Severity Scale (FSS) and gave written consent prior to blood collection. Ethics approval was granted by the Griffith University Human Research Ethics Committee, reference number (HREC/15/QGC/63).

\section{Isolation of human NK cells}

Isolation of NK cells was conducted as previously described..$^{10}$ Briefly, $30 \mathrm{~mL}$ of EDTA blood was incubated with $1.5 \mathrm{~mL}$ of NK enrichment cocktail (Stem Cell Technologies, USA) for 25 minutes at room temperature. Blood was then diluted with $30 \mathrm{~mL}$ of unsupplemented RPMI and layered over Ficoll-plaque, prior to centrifugation at $1200 \mathrm{~g}$ for 20 minutes at $20^{\circ} \mathrm{C}$. The enrichment cell layer was removed and pelleted at $400 \mathrm{~g}$ for 10 minutes at $4^{\circ} \mathrm{C}$. NK cells were washed in pre-chilled unsupplemented RPMI to ensure metabolic state was preserved of NK cells.

\section{Viability and purity of NK cells}

NK cell suspension and trypan blue stain at a ratio 1:1 was used to measure viability. Viability was calculated using a Biorad automatic cell counter (Supplementary Table 1). A final concentration of $2 \times 10^{5} \mathrm{NK}$ cells was stained with antiCD56-APC and anti-CD3-BV510 antibodies and incubated

Supplement Table 1 . Viability of NK cells from healthy control and ME/CFS patients.

\begin{tabular}{lccc}
\hline Viability $(\% \pm \mathrm{SD})$ & Healthy control & ME/CFS & $\boldsymbol{P}$-value \\
\hline Mitostress & $88.40 \pm 6.81$ & $89.75 \pm 4.97$ & 0.83 \\
Glycolysis stress & $83.22 \pm 12.28$ & $88.85 \pm 6.46$ & 0.14 \\
\hline
\end{tabular}


for 25 minutes. Purity was measured using LSR Fortessa X-20 flow cytometry cell analyzer. Gating strategy to identify NK cells and check for purity was defined as the percentage of CD56 ${ }^{+} \mathrm{CD} 3^{-}$cells. NK cell purity was $91.83 \% \pm 1.65$ and $93.55 \%$ \pm 1.47 in non-fatigued healthy control and ME/CFS, respectively (Supplement Figure 1).

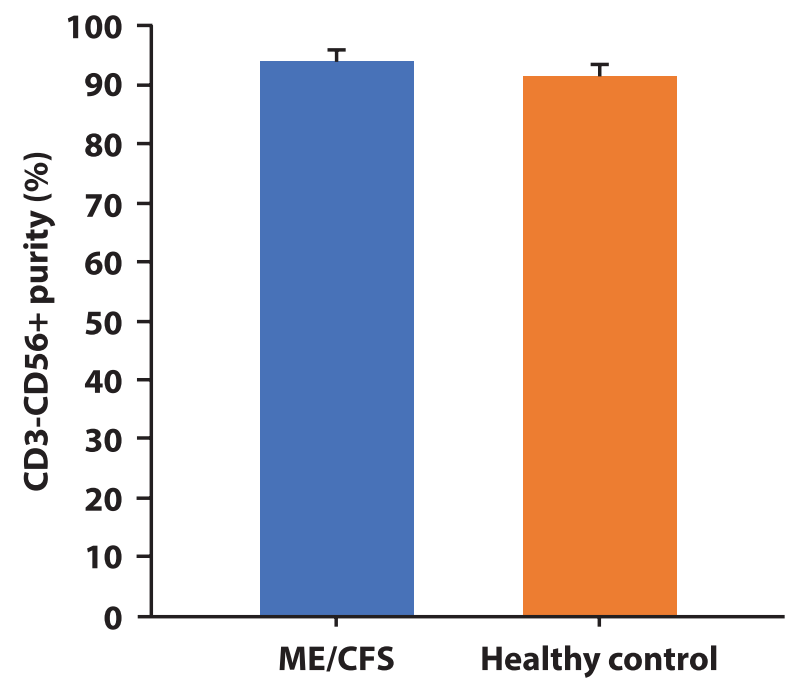

Supplement Figure 1.

\section{Immobilised NK cells to XF cell culture microplate}

XF cell culture plate was coated with Cell-Tak as per manufacturer's instructions. Briefly, a final concentration of 22.4 $\mathrm{ug} / \mathrm{mL}$ Cell-Tak solution (Cell-Tak, sodium bicarbonate, and sodium hydroxide) was added to each well for 30 minutes at room temperature. Wells were washed twice with $200 \mu \mathrm{L}$ of distilled-tissue culture grade water (Gibco) and air dried in the biosafety cabinet. NK cells were seeded at a final concentration of $2 \times 10^{5}$ cells/well in either mito or glycolysis stress test medium on the Cell-Tak coated plate and samples were plated in triplets. Plates were gently shaken to assist the cells to settle evenly for 5 minutes at room temperature. Plates were centrifuged twice at $200 \mathrm{~g}$ for 1 minute in $4^{\circ} \mathrm{C}$. After each centrifugation, the plate was re-orientated to maintain cell density. Plates were washed with either pre-chilled mito or glycolysis assay media (as manufacture's instruction) and the cells were resuspended in a final volume of $180 \mu \mathrm{L}$. The plate was returned to the centrifuge at $200 \mathrm{~g}$ for 1 minute in $4^{\circ} \mathrm{C}$ and viewed under a microscope to assess cells detachment and density. The plate was incubated for 15 minutes in a non- $\mathrm{CO}_{2}$ incubator, prior to acquisition on Seahorse XFp Analyser. Mitochondrial stress assay was analyzed first, followed by glycolysis. NK cells used for the glycolysis assay were placed in $4^{\circ} \mathrm{C}$ to prevent metabolic activation.

\section{Mitochondrial stress assay}

XF Mito stress test (Seahorse Bioscience, North Billerica, MA, USA) was performed following the manufacturer's instruction. ${ }^{21}$ Briefly, NK cells are immobilised within the XF base medium supplemented with a final concentration of 10 $\mathrm{mM}$ glucose, $1 \mathrm{mM}$ sodium pyruvate and $2 \mathrm{mM}$ L-glutamine. Medium was warmed in a water bath at $37^{\circ} \mathrm{C}$, before $\mathrm{pH}$ adjustment to $7.4 \pm 0.1$ using $1 \mathrm{~N} \mathrm{NaOH}$. The baseline was recorded using four measurements, prior to an injection of a final concentration of $1 \mu \mathrm{M}$ oligomycin. Oligomycin inhibits ATP production from the mitochondrial respiration. Following, $1 \mu \mathrm{M}$ of Carbonyl cyanide-4 (trifluoromethoxy) phenylhydrazone (FCCP) resulting in the disruption of the proton gradient and mitochondrial membrane potential (Supplement Figure 2). Lastly, mitochondrial respiration was inhibited after an injection of $0.5 \mu \mathrm{M}$ Rotenone and Antimycin mixture, which are a complex I and a complex III inhibitor, respectively. Mitochondrial respiration has been reported as oxygen consumption rate (OCR) at $\mathrm{pmol} / \mathrm{min} .{ }^{21}$ Bioenergetic health index (BHI) was used to measure the NK cells overall mitochondrial biogenetic function and was calculated based on the formula below. ${ }^{22}$

$\mathrm{BHI}=\log \frac{(\text { spare repiratory capacity }) \times(A T P \text { production })}{(\text { non-mitochondria repiration }) \times(\text { proton leak })}$

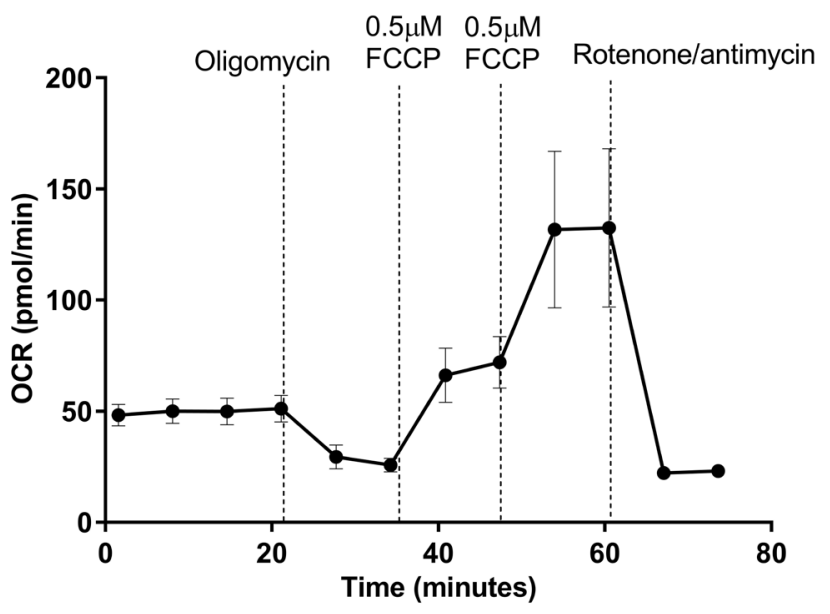

Supplement Figure 2.

\section{Glycolysis stress assay}

XF Glycolysis stress test (Seahorse Bioscience, North Billerica, MA, USA) measured glycolysis, glycolytic capacity and glycolytic reserve as per manufacturer's instructions. ${ }^{21}$ Briefly, NK cells were immobilised in Glycolytic stress medium was comprised of XF base medium supplemented with a final concentration of $2 \mathrm{mM}$ L-glutamine. $\mathrm{pH}$ was adjusted to $7.4 \pm$ 0.1 using $1 \mathrm{~N} \mathrm{NaOH}$ in a $37^{\circ} \mathrm{C}$ water bath. Baseline values were recorded prior to a final concentration of $10 \mu \mathrm{M}$ glucose injection. NK cells utilise the glucose for glycolysis and produce pyruvate, ATP, NADH and protons. Subsequently, a final concentration of $1 \mu \mathrm{M}$ oligomycin was injected to inhibit Complex V, an ATP synthase. Oligomycin inhibits ATP production from mitochondrial respiration, which increases cellular energetic demands and shifts energy production to glycolysis. Lastly, a final concentration of $50 \mathrm{mM}$ 2-Deoxy -D-glucose (2-DG) was injected, which inhibits glycolysis through competitively binding to the first enzyme in the glycolytic pathway, glucose hexokinase. Glycolytic parameters were reported as Extracellular Acidification Rate (ECAR) at $\mathrm{mpH} / \mathrm{min}$.

\section{Statistical data analysis}

Wave data were converted into excel format prior to performing analyses on the XF report generators. XF mito stress 
and XF glycolysis stress test report generator automatically calculated mitochondrial respiration and glycolytic test parameters with background correction. XF mito stress test result was also analyzed in the BHI test report generator for overall mitochondrial bioenergetic function in isolated NK cells. Statistical analyses were performed in SPSS Version 22. Shapiro-Wilk analysis and Leven's test were used to determine normality and homogeneity. One-way ANOVA was used to determine demographic characteristics and blood parameters in healthy controls and ME/CFS. One-way ANOVA was also used to determine the difference in mitochondrial respiration or glycolytic function in NK cells between healthy controls compared with ME/CFS patients. All data were represented as the mean \pm standard deviation (SD), and statistical significance was reported as $P<0.05$ (Figure 2) and $P<0.01$ (Table 1).

\section{Results}

The percentage of male to female participants $(16.66 \%$ and $83.33 \%$, respectively) was the same in both healthy controls and ME/CFS group. There was no significant difference between groups for subject characteristics or full blood count parameters (Table 1). All participants completed a Fatigue Severity Scale (FSS) of 1 to 7, with higher scores indicating greater disability. The ME/CFS group scored significantly higher than the healthy control group ( $5.51 \pm 0.66$ vs. $1.37 \pm 0.34)$.

\section{Resting NK cells' mitochondrial respiration was not statistical- ly different between groups}

Oxygen consumption rate in NK cells from both groups was not significantly different when exposed to oligomycin, FCCP, Rotenone, and antimycin A (Figure 1A). Oligomycin was used to determine cellular ATP production by inhibiting ATP synthase (complex V) in the ETC. FCCP disrupted the mitochondrial membrane potential and proton gradient resulting in increased oxygen consumption by complex V. Spare respiratory capacity was determined by the difference between basal ATP production and the maximal ability the respiratory
Table 1.

\begin{tabular}{|c|c|c|c|}
\hline $\begin{array}{l}\text { Demographic } \\
\text { parameter }\end{array}$ & $\begin{array}{c}\text { Control } \\
(\mathbf{n}=6)\end{array}$ & $\underset{(\mathrm{n}=6)}{\mathrm{ME} / \mathrm{CFS}}$ & $P$-Value \\
\hline Sex & & & N/A \\
\hline Female & $83.33 \%$ & $83.33 \%$ & \\
\hline Male & $16.66 \%$ & $16.66 \%$ & \\
\hline Age & $50.00 \pm 5.04$ & $50.33 \pm 4.95$ & 0.963 \\
\hline FSS Score & $1.37 \pm 0.34$ & $5.51 \pm 0.66$ & $0.004^{*}$ \\
\hline \multicolumn{4}{|l|}{ Blood parameters } \\
\hline \multicolumn{4}{|l|}{ Leukocytes } \\
\hline White Cell Count $\left(\times 10^{9} / \mathrm{L}\right)$ & $6.25 \pm 1.12$ & $5.93 \pm 1.13$ & 0.638 \\
\hline Lymphocytes $\left(\times 10^{9} / \mathrm{L}\right)$ & $2.16 \pm 0.59$ & $2.11 \pm 0.34$ & 0.858 \\
\hline Monocytes $\left(\times 10^{9} / \mathrm{L}\right)$ & $0.30 \pm 0.05$ & $0.28 \pm 0.03$ & 0.407 \\
\hline Neutrophils $\left(\times 10^{9} / \mathrm{L}\right)$ & $3.62 \pm 0.96$ & $3.38 \pm 1.13$ & 0.705 \\
\hline Eosinophils $\left(\times 10^{9} / \mathrm{L}\right)$ & $0.13 \pm 0.03$ & $0.11 \pm 0.08$ & 0.704 \\
\hline Basophils $\left(\times 10^{9} / \mathrm{L}\right)$ & $0.02 \pm 0.01$ & $0.02 \pm 0.01$ & 0.451 \\
\hline Platelets $\left(\times 10^{9} / \mathrm{L}\right)$ & $277.66 \pm 49.11$ & $314.66 \pm 53.59$ & 0.241 \\
\hline \multicolumn{4}{|l|}{ Erythrocytes } \\
\hline Haemoglobin $(\mathrm{g} / \mathrm{L})$ & $135.33 \pm 19.57$ & $134.5 \pm 10.98$ & 0.929 \\
\hline Haematocrit (\%) & $0.40 \pm 0.04$ & $0.40 \pm 0.02$ & 1.00 \\
\hline Red Cell Count $\left(\times 10^{12} / \mathrm{L}\right)$ & $4.60 \pm 0.57$ & $3.97 \pm 1.48$ & 0.359 \\
\hline $\begin{array}{l}\text { Mean Corpuscular Volume } \\
\text { (fL) }\end{array}$ & $87.83 \pm 3.06$ & $90.16 \pm 2.99$ & 0.212 \\
\hline \multicolumn{4}{|l|}{ Electrolytes } \\
\hline Sodium $(\mathrm{mmol} / \mathrm{L})$ & $138.00 \pm 1.41$ & $136.16 \pm 2.63$ & 0.165 \\
\hline Potassium $(\mathrm{mmol} / \mathrm{L})$ & $4.03 \pm 0.24$ & $4.15 \pm 0.27$ & 0.453 \\
\hline Chloride $(\mathrm{mmol} / \mathrm{L})$ & $101.66 \pm 3.01$ & $101.00 \pm 2.89$ & 0.704 \\
\hline Bicarbonate $(\mathrm{mmol} / \mathrm{L})$ & $28.50 \pm 2.07$ & $27.83 \pm 2.22$ & 0.603 \\
\hline Anion Gap (mmol/L) & $7.66 \pm 1.36$ & $7.33 \pm 1.03$ & 0.644 \\
\hline \multicolumn{4}{|l|}{ Inflammatory markers } \\
\hline $\begin{array}{l}\text { Erythrocyte sedimentation } \\
\text { rate }(\mathrm{mm} / \mathrm{Hr})\end{array}$ & $11.50 \pm 5.46$ & $19.50 \pm 14.40$ & 0.232 \\
\hline $\begin{array}{l}\text { High sensitive C-Reactive } \\
\text { Protein }(\mathrm{mg} / \mathrm{mL})\end{array}$ & $1.08 \pm 0.59$ & $2.63 \pm 3.75$ & 0.341 \\
\hline
\end{tabular}

A.

Mito stress
B.

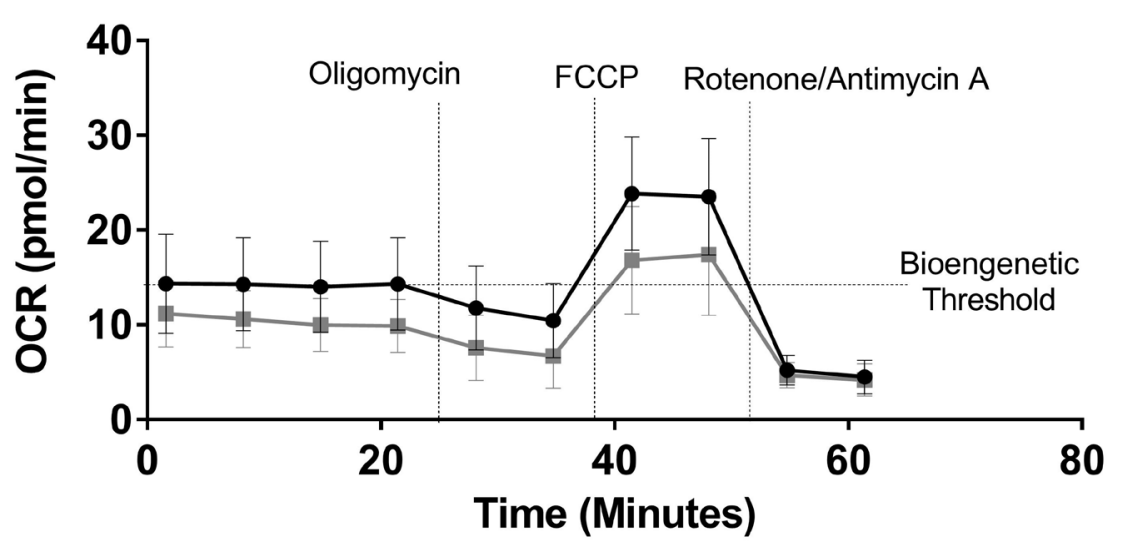

$\rightarrow$ Control $\rightarrow$ CFS/ME

\section{Basal respiration}

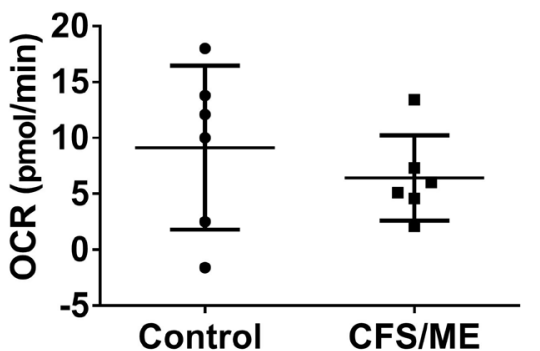

Figure 1. Mitostress 
C.

Proton leak

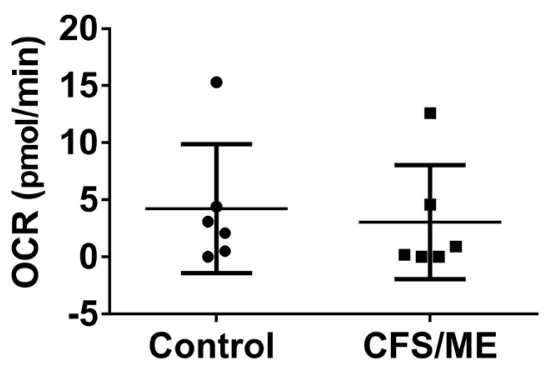

F.

Spare respiration capacity

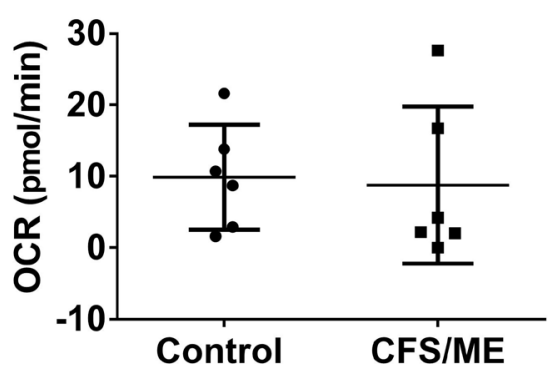

D.

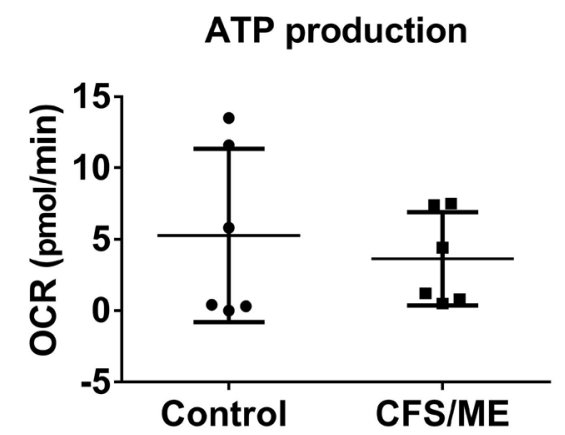

G.
E.

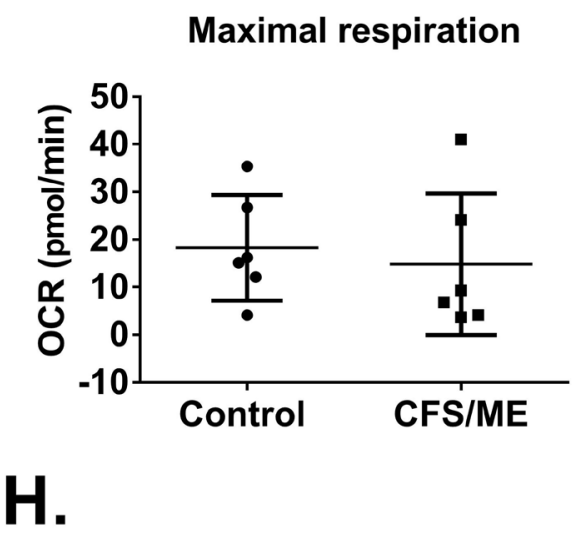

Bioenergetic Health Index
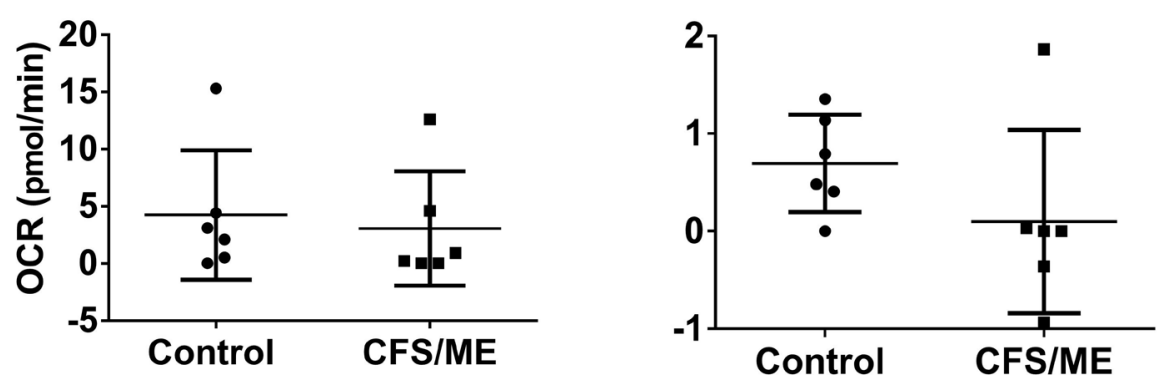

Figure 1. (Continued)

chain operates under energy demands. This indicates the capability for NK cells to respond to energy demands and ability to overcome mitochondrial stress. Rotenone and antimycin A were exposed to NK cells to inhibit complex I and complex III, respectively. This combination of drugs inhibits mitochondrial respiration and helps define non-mitochondrial respiration, i.e., background noise of oxygen molecules not produced from mitochondrial respiration. There was no significant difference

A.

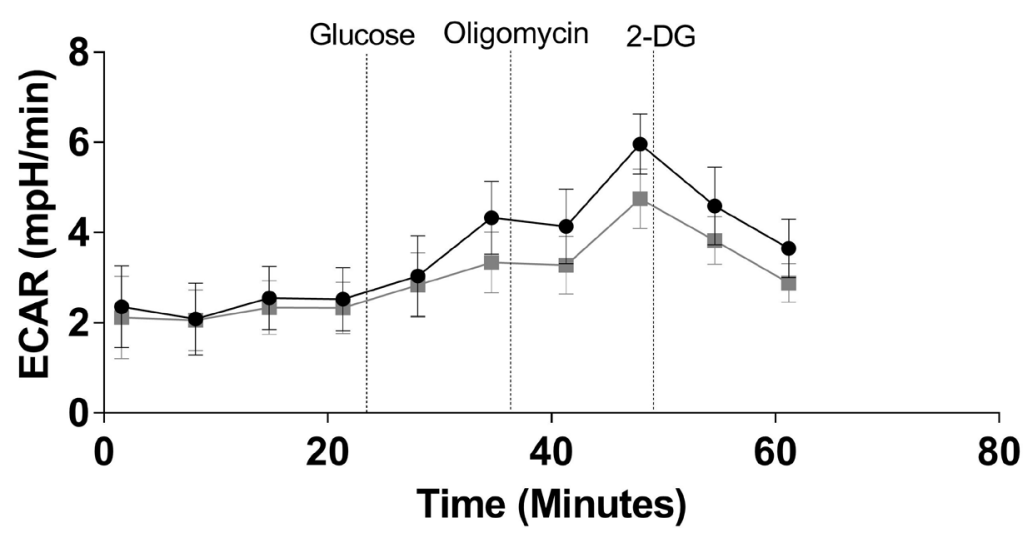

- Control $-\mathrm{ME} / \mathrm{CFS}$ observed in mitochondrial respiration function such as basal respiration (Figure 1B), proton leak (Figure 1C), ATP production (Figure 1D), maximum respiration (Figure 1E), spare respiratory capacity (Figure 1F) and coupling efficiency (Figure 1G) in resting NK cells from healthy controls compared with $\mathrm{ME} / \mathrm{CFS}$ group. BHI score of the bioenergetics profile in isolated NK cells from ME/CFS and healthy control group did not reach significance (Figure $\mathbf{1 H}$ ).
B.

non-glycolytic acidification

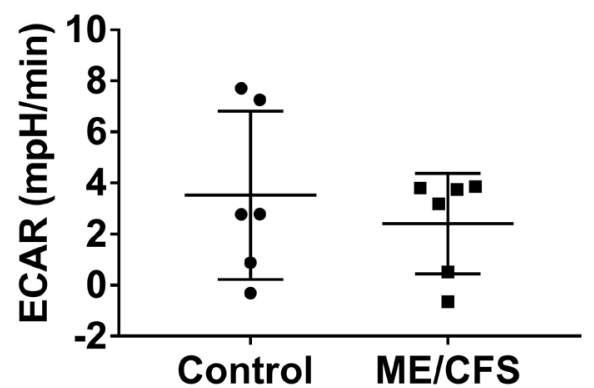

Figure 2. Glycolysis stress 
C.

\section{Glycolysis}

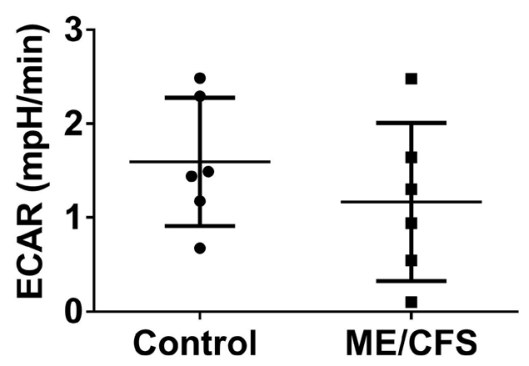

D.

Glycolytic Capacity

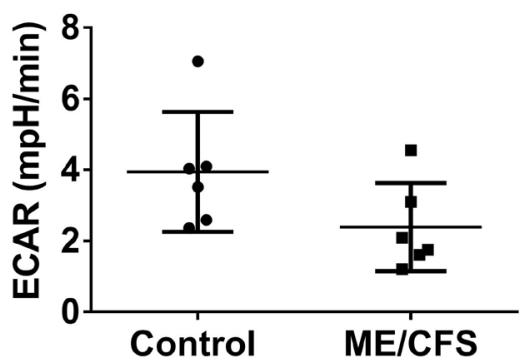

Glycolytic reserve

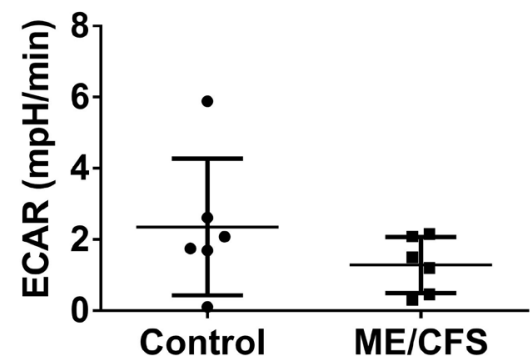

Figure 2. (Continued)

\section{Reduced glycolytic reserve identified in resting NK cells from ME/CFS patients}

Extracellular acidification rates measured NK cells' glycolytic functions (including glycolytic reserve, glycolysis, and glycolytic capacity) during exposure to glucose, oligomycin and 2-DG in healthy control and ME/CFS patients (Figure 2A). The glycolytic reserve was the measurement of the NK cells' ability to increase glycolytic flux in response to high energetic demands for ATP production, which was determined by the presence and absence of oligomycin. The glycolytic reserve was significantly decreased in NK cells from ME/CFS patients compared with healthy controls (Figure 2B). Glycolysis is the measurement of the NK cells' glycolytic activity in the presence of glucose. Glycolysis was higher in ME/CFS compared to healthy controls but did not reach statistical significance (Figure 2C). Glycolytic capacity is the measurement of NK cells' capability of producing ATP by glycolysis. There was no significant difference between groups for glycolytic capacity for resting NK cells (Figure 2D).

\section{Discussion}

This preliminary investigation is the first to report a significant reduction of glycolytic reserve in resting isolated NK cells from ME/CFS patients compared with aged-matched non -fatigued healthy controls. The novel finding may perhaps postulate NK cells from ME/CFS may have difficulty upregulating glycolysis for effector function in response to immune challenges or during stress.

Rapamycin complex 1 (mTORC1) is essential for maintaining high glycolytic state in activated NK cells. Disruption of glycolysis inhibits production of effector molecules such as granzyme B and IFN- $\gamma \cdot{ }^{17}$ The mTOR signaling pathway is regulated by RPS6KA2 and SGK1, which has shown significant hypomethylation in ME/CFS patients. ${ }^{23}$ MTOR activates a transcription factor known as hypoxia-inducible factor 1 (HIF1). HIF1a decreases glycolytic reserve through decreasing ETC complex I and II function and increasing ROS from both ETC and mitochondria, which subsequently decrease ATP production. ${ }^{24}$ Hence, reduced glycolytic reserve hinders energy production for cytokines and lytic protein synthesis. ${ }^{17}$ Importantly, significant decreases in IFN- $\gamma$, perforin and granzyme $\mathrm{B}$ from ME/CFS patients may explain the reduced CD56 ${ }^{\text {Dim }}$ NK cytotoxic activity and the decreased IFN- $\gamma$ production in
CD56 ${ }^{\text {Bright }}$ NK cells. ${ }^{11,15,16}$ Without IFN- $\gamma$ production, CD56 ${ }^{\text {Bright }}$ NK cells cannot effectively recruit dendritic and T cells to the site of inflammation. ${ }^{25}$

Glycolytic reserve level is dependent on NK cells' maximum glycolytic flux and glycolysis to respond to an energetic demand. However, glycolysis was lower in ME/CFS compared to healthy controls but did not reach statistical significance. Glycolysis is required for the biosynthesis of the amino acid pathway to produce arginine. ${ }^{26}$ In particular, L-Arginine is combined with co-factors (including NADPH, FAD, BH4, Haem, and calmodulin) to produce nitric oxide and oxygen molecules via nitric oxide synthase. Nitric oxide (NO) and oxygen $\left(\mathrm{O}_{2}\right)$ can form peroxynitrite $\left(\mathrm{ONOO}^{-}\right)$, and these levels have been reported to be elevated in ME/CFS patients' blood. ${ }^{27}$ Furthermore, decreased NO-mediated NK cell activation has been reported in ME/CFS patients. ${ }^{28} \mathrm{NO}$ mediates L-Arg-induced activation of NK activity and that a possible dysregulated in the glycolytic metabolic pathway. ${ }^{28,29}$ Although extracellular Arg was not significantly different between ME/CFS and healthy control, the role of intracellular role of Arg in NK cells remains unclear. ${ }^{30}$

The glycolytic function is also regulated by glycolytic substrate levels, such as ATP and citrate, which inhibit glycolysis via phosphofructokinase. Citrate is produced from the substrates oxaloacetate and acetyl CoA via citrate synthase. Interestingly, reduced citrate synthase activity has been reported in ME/CFS patients. ${ }^{4,31}$ A reduction in citrate provides for the loss of allosteric regulation to inhibit glycolysis and facilitate glucose to be re-directed to the pentose phosphate pathway for NADPH production, which is essential for fatty acid synthesis. This pathway also compromises the availability of glutathione to remove hydrogen peroxide and may contribute to the evaluation of free radical, low fatty acids and high oxidized LDL antibodies reported in ME/CFS patients. ${ }^{32}$ Alternatively, reduced glycolytic reserve in combination with reduced citrate synthase activity may promote pyruvate to be converted into lactate and removed from the cell.,19,31 This is essential as the accumulation of pyruvate may result in inhibition of glycolysis. $\mathrm{ME} / \mathrm{CFS}$ patients have reported increased blood lactate, muscular lactate and ventricular lactate. ${ }^{33}$

In the present study mitochondrial respiration was not significantly different in both age-matched healthy controls and ME/CFS groups. Whereas, Tomas et al (2017) showed ME/CFS showed significant reduced mitochondria respiration in serum free isolated PBMC. Perhaps PBMC which contains different 
cell populations (monocytes, T cells, B cells and NK cells) have a slightly different serum free microenvironment compare with serum free isolated NK cells, which may impact on metabolism. Alternatively, other studies have shown ME/CFS reduced mitochondria respiration drives glycolysis to compensate for high energy demands. ${ }^{9,30}$ Perhaps NK cell may not require glycolysis to compensate for the increased in energy demand, as the mitochondria respiration was the same in ME/CFS and healthy control. This may suggest that the increased in lactate production by other cells (ie, muscle cells or tumor cells) into the microenvironment may influence NK cell effector function and alternative the metabolism. For instance, lactate downregulated NKp46 receptors on NK cells and reduce production of lytic proteins. ${ }^{34}$ Therefore, the microenvironment may alter the metabolic phenotype of NK cells in ME/CFS patients.

A major limitation to this study is sample size and a larger cohort is warranted to confirm these finding. Although glycolytic reserve in resting NK cells is reduced in ME/CFS it is unclear whether the cause of this reduction is intracellular or extracellular. A previous study has identified reduced amino acids in serum of ME/CFS patients and that the microenvironment play a key role in how immune cells response under high energy demands. ${ }^{30}$ Further comparison investigation of NK cells glycolytic function in serum and serum free medium may provide further insight on the effects of systematic factors (such as lactate, glucose and amino acids) on NK cells metabolism in $\mathrm{ME} / \mathrm{CFS}$.

This preliminary study has demonstrated a significant reduction in glycolytic reserves in isolated NK cells from ME/CFS patients. Our findings suggest impaired glycolytic metabolic pathways in response to high energetic demand. Glycolysis has been shown to be one of the major energy-producing pathways that feed into other metabolic pathways, which may explain the multi-systemic nature of ME/CFS. Hence, this study may contribute to future metabolic investigations for challenged or stimulated isolated NK cells from ME/CFS patients to determine NK cells' bioenergetic profile in the active state.

\section{Acknowledgements}

This study was supported by funding from the Stafford Fox Medical Research Foundation, the Alison Hunter Memorial Foundation, Mason Foundation, Mr Douglas Stutt, Blake Beckett Foundation and Queensland Co-Investment Program.

\section{Conflict of interest}

Authors do not have any conflict of interest

\section{Author's contribution}

Nguyen contributed to the overall experimental design, assist in experimental set-up and data acquisition, analysis and draft the manuscript. Johnston contributed to participant's demographics, serological and drafting process. Marshall-Gradisnik, and Staines contributed to the concept, drafting and revising of the paper. All authors read and approved the final manuscript.

\section{References}

1. Carruthers BM, van de Sande MI, De Meirleir KL, Klimas NG, Broderick G, Mitchell T, et al. Myalgic encephalomyelitis: International Consensus Criteria. J Intern Med. 2011;270:327-38.

2. Booth NE, Myhill S, McLaren-Howard J. Mitochondrial dysfunction and the pathophysiology of Myalgic Encephalomyelitis/Chronic Fatigue Syndrome (ME/CFS). Int J Clin Exp Med. 2012;5:208-20.

3. Morris G, Maes M. Mitochondrial dysfunctions in myalgic encephalomyelitis/chronic fatigue syndrome explained by activated immuno-inflammatory, oxidative and nitrosative stress pathways. Metab Brain Dis. 2014;29:19-36.

4. Smits B, van den Heuvel L, Knoop H, Kusters B, Janssen A, Borm G, et al. Mitochondrial enzymes discriminate between mitochondrial disorders and chronic fatigue syndrome. Mitochondrion. 2011;11:735-8.

5. Jammes Y, Steinberg JG, Mambrini O, Bregeon F, Delliaux S. Chronic fatigue syndrome: assessment of increased oxidative stress and altered muscle excitability in response to incremental exercise. J Intern Med. 2005;257:299-310

6. Germain A, Ruppert D, Levine SM, Hanson MR. Metabolic profiling of a myalgic encephalomyelitis/chronic fatigue syndrome discovery cohort reveals disturbances in fatty acid and lipid metabolism. Mol Biosyst. 2017;13:371-9.

7. Nagy-Szakal D, Williams BL, Mishra N, Che X, Lee B, Bateman L, et al. Fecal metagenomic profiles in subgroups of patients with myalgic encephalomyelitis/chronic fatigue syndrome. Microbiome. 2017;5:44.

8. Naviaux RK, Naviaux JC, Li K, Bright AT, Alaynick WA, Wang L, et al. Metabolic features of chronic fatigue syndrome. Proc Natl Acad Sci U S A. 2016;113:E5472-80.

9. Tomas C, Brown A, Strassheim V, Elson J, Newton J, Manning P. Cellular bioenergetics is impaired in patients with chronic fatigue syndrome. Plos One. 2018;13:e0192817.

10. Huth TK, Brenu EW, Ramos S, Nguyen T, Broadley S, Staines D, et al. Pilot Study of Natural Killer Cells in Chronic Fatigue Syndrome/Myalgic Encephalomyelitis and Multiple Sclerosis. Scand J Immunol. 2016;83: 44-51.

11. Maher KJ, Klimas NG, Fletcher MA. Chronic fatigue syndrome is associated with diminished intracellular perforin. Clinical and experimental immunology. 2005;142:505-11.

12. Morris G, Anderson G, Galecki P, Berk M, Maes M. A narrative review on the similarities and dissimilarities between myalgic encephalomyelitis/ chronic fatigue syndrome (ME/CFS) and sickness behavior. BMC Med. 2013;11:64.

13. Poli A, Michel T, Theresine M, Andres E, Hentges F, Zimmer J. CD56bright natural killer (NK) cells: an important NK cell subset. Immunology. 2009; 126:458-65.

14. Keppel MP, Saucier N, Mah AY, Vogel TP, Cooper MA. Activation-specific metabolic requirements for NK Cell IFN-gamma production. Journal of immunology (Baltimore, Md : 1950). 2015;194:1954-62.

15. Brenu EW, van Driel ML, Staines DR, Ashton KJ, Hardcastle SL, Keane J, et al. Longitudinal investigation of natural killer cells and cytokines in chronic fatigue syndrome/myalgic encephalomyelitis. J Transl Med.2012; 10:88.

16. Brenu EW, van Driel ML, Staines DR, Ashton KJ, Ramos SB, Keane J, et al. Immunological abnormalities as potential biomarkers in Chronic Fatigue Syndrome/Myalgic Encephalomyelitis. J Transl Med. 2011;9:81.

17. Donnelly RP, Loftus RM, Keating SE, Liou KT, Biron CA, Gardiner CM, et al. mTORC1-dependent metabolic reprogramming is a prerequisite for NK cell effector function. Journal of immunology (Baltimore, Md : 1950). 2014;193:4477-84.

18. Frauwirth KA, Riley JL, Harris MH, Parry RV, Rathmell JC, Plas DR, et al. The CD28 signaling pathway regulates glucose metabolism. Immunity. 2002;16:769-77.

19. Finlay DK. Metabolic regulation of natural killer cells. Biochem Soc Trans. 2015;43:758-62.

20. Fukuda K, Straus SE, Hickie I, Sharpe MC, Dobbins JG, Komaroff A. The chronic fatigue syndrome: a comprehensive approach to its definition and study. International Chronic Fatigue Syndrome Study Group. Ann Intern Med. 1994;121:953-9.

21. Pelletier M, Billingham LK, Ramaswamy M, Siegel RM. Extracellular Flux Analysis to Monitor Glycolytic Rates and Mitochondrial Oxygen Consumption. Method Enzymol. 2014;542:125-49. 
APJAI

22. Chacko BK, Kramer PA, Ravi S, Benavides GA, Mitchell T, Dranka BP, et al. The Bioenergetic Health Index: a new concept in mitochondrial translational research. Clin Sci (Lond). 2014;127:367-73.

23. Brenu EW, Marshall-Gradisbik S, Staines D. Methylation Profile of CD4+ T Cells in Chronic Fatigue Syndrome/Myalgic Encephalomyelitis. J Clin Cell Immunol. 2014,5.

24. Semenza GL. HIF-1 mediates metabolic responses to intratumoral hypoxia and oncogenic mutations. J Clin Invest. 2013;123:3664-71.

25. Ge MQ, Ho AW, Tang Y, Wong KH, Chua BY, Gasser S, et al. NK cells regulate $\mathrm{CD} 8+\mathrm{T}$ cell priming and dendritic cell migration during influenza A infection by IFN-gamma and perforin-dependent mechanisms. Journal of immunology (Baltimore, Md : 1950). 2012;189:2099-109.

26. Luiking YC, Ten Have GAM, Wolfe RR, Deutz NEP. Arginine de novo and nitric oxide production in disease states. Am J Physiol-Endoc M. 2012; 303:E1177-E89.

27. Pall ML, Satterlee JD. Elevated nitric oxide/peroxynitrite mechanism for the common etiology of multiple chemical sensitivity, chronic fatigue syndrome, and posttraumatic stress disorder. Ann N Y Acad Sci. 2001; 933:323-9.

28. Ogawa M, Nishiura T, Yoshimura M, Horikawa Y, Yoshida H, Okajima Y, et al. Decreased nitric oxide-mediated natural killer cell activation in chronic fatigue syndrome. Eur J Clin Invest. 1998;28:937-43.
29. Jyothi MD, Khar A. Induction of nitric oxide production by natural killer cells: its role in tumor cell death. Nitric Oxide. 1999;3:409-18.

30. Fluge O, Mella O, Bruland O, Risa K, Dyrstad SE, Alme K, et al. Metabolic profiling indicates impaired pyruvate dehydrogenase function in myalgic encephalopathy/chronic fatigue syndrome. JCI Insight. 2016;1:e89376.

31. McArdle A, McArdle F, Jackson MJ, Page SF, Fahal I, Edwards RH Investigation by polymerase chain reaction of enteroviral infection in patients with chronic fatigue syndrome. Clin Sci (Lond). 1996;90:295-300.

32. Maes $M$, Kubera $M$, Uytterhoeven $M$, Vrydags $N$, Bosmans $E$. Increased plasma peroxides as a marker of oxidative stress in myalgic encephalomyelitis/chronic fatigue syndrome (ME/CFS). Med Sci Monit. 2011;17:SC11-5.

33. Vermeulen RC, Kurk RM, Visser FC, Sluiter W, Scholte HR. Patients with chronic fatigue syndrome performed worse than controls in a controlled repeated exercise study despite a normal oxidative phosphorylation capacity. J Transl Med. 2010;8:93.

34. Scott KE, Cleveland JL. Lactate Wreaks Havoc on Tumor-Infiltrating T and NK Cells. Cell Metab. 2016;24:649-50. 